\title{
DGK Herztage 2021
}

30.09.-02.10.2021

๑) Springer-Verlag GmbH Germany, part of Springer Nature 2021

Deutsche Gesellschaft für Kardiologie

- Herz- und Kreislaufforschung e.V.

Tagungspräsident*innen / Presidents of the conference:

Tagungspräsident Kardiologie Aktuell 2021

Dr. Norbert Smetak, Kirchheim/Teck

Tagungspräsidentin Deutsche Rhythmus Tage 2021

Prof. Dr. Isabel Deisenhofer, München

Tagungspräsident AGIKlive 2021

Prof. Dr. Helge Möllmann, Dortmund

\section{Organisatoren Akademiekurse}

Prof. Dr. Norbert Frey, Heidelberg

Prof. Dr. Albrecht Elsässer, Oldenburg
Organisatoren Basic Science Meeting

Prof. Dr. Dierk Thomas, Heidelberg

Dr. Malte Tiburcy, Göttingen

\begin{abstract}
s
Dieser Link führt Sie zur Abstracts-Datenbank, in der Sie per Schlagwortsuche online nach Dokumenten suchen können.

This link leads you to the searchable online abstract data base.

https://dgk.org/kongress_programme/ht2021/abstracts/
\end{abstract}

